\title{
Cortical inactivation by cooling in small animals
}

\author{
Ben Coomber ${ }^{1}$, Darren Edwards' ${ }^{1}$, Simon J. Jones ${ }^{1}$, Trevor M. Shackleton ${ }^{1}$, Jürgen Goldschmidt ${ }^{2}$, \\ Mark N. Wallace ${ }^{1}$ and Alan R. Palmer ${ }^{*}$
}

1 MRC Institute of Hearing Research, University Park, Nottingham, UK

2 Leibniz Institut für Neurobiologie, Magdeburg, Germany

\section{Edited by:}

Federico Bermudez-Rattoni,

Universidad Nacional Autónoma de

México, Mexico

\section{Reviewed by:}

Milagros Gallo, University of Granada, Spain

Takashi Yamamoto, Osaka University Graduate School of Dentistry, Japan

\section{${ }^{*}$ Correspondence}

Alan R. Palmer, MRC Institute of Hearing Research, Nottingham

University Section, University Park,

Nottingham NG7 2RD, UK.

e-mail:alan.palmer@ihr.mrc.ac.uk
Reversible inactivation of the cortex by surface cooling is a powerful method for studying the function of a particular area. Implanted cooling cryoloops have been used to study the role of individual cortical areas in auditory processing of awake-behaving cats. Cryoloops have also been used in rodents for reversible inactivation of the cortex, but recently there has been a concern that the cryoloop may also cool non-cortical structures either directly or via the perfusion of blood, cooled as it passed close to the cooling loop. In this study we have confirmed that the loop can inactivate most of the auditory cortex without causing a significant reduction in temperature of the auditory thalamus or other subcortical structures. We placed a cryoloop on the surface of the guinea pig cortex, cooled it to $2^{\circ} \mathrm{C}$ and measured thermal gradients across the neocortical surface. We found that the temperature dropped to $20-24^{\circ} \mathrm{C}$ among cells within a radius of about $2.5 \mathrm{~mm}$ away from the loop. This temperature drop was sufficient to reduce activity of most cortical cells and led to the inactivation of almost the entire auditory region. When the temperature of thalamus, midbrain, and middle ear were measured directly during cortical cooling, there was a small drop in temperature (about $4^{\circ} \mathrm{C}$ ) but this was not sufficient to directly reduce neural activity. In an effort to visualize the extent of neural inactivation we measured the uptake of thallium ions following an intravenous injection. This confirmed that there was a large reduction of activity across much of the ipsilateral cortex and only a small reduction in subcortical structures.

Keywords: auditory cortex, cooling inactivation, cryoloop, thallium autometallography

\section{INTRODUCTION}

A significant challenge in auditory neuroscience lies in understanding the descending projections from auditory cortex to subcortical auditory nuclei, and how these projections shape the processing of auditory information. A variety of anatomical and electrophysiological studies have demonstrated the existence of descending control from auditory cortex to the medial geniculate body (MGB; Kelly and Wong, 1981; He, 2003), inferior colliculus (IC; Saldaña et al., 1996; Coomes Peterson and Schofield, 2007; Nakamoto et al., 2008), and the cochlear nucleus (Feliciano and Potashner, 1995; Weedman and Ryugo, 1996a,b; Luo et al., 2008; Liu et al., 2010), but the functional significance of such projections is poorly understood.

Cortical inactivation is a useful approach for determining the extent of modulation by descending projections. There are a number of ways in which to inactivate cortex that differ in reversibility, magnitude, and duration of sustained effects. The cryoloop cooling technique for cortical inactivation produces a reversible, highly localized inhibition of neuronal activity when the temperature of neurones is reduced to $<20^{\circ} \mathrm{C}$ (Lomber et al., 1994, 1996, 1999). We have recently been using the cryoloop technique to study corticofugal modulation in the auditory system of guinea pigs, by inactivating auditory cortex and examining changes in the firing pattern of IC neurones in response to sounds (Nakamoto et al., 2008).

Abbreviations: CSD, current source-density; Cx, neocortex; DDC, diethyldithiocarbamate; GP, guinea pig; H, hippocampus; IC, inferior colliculus; MCA, middle cerebral artery; MGB, medial geniculate body; RMS, root mean squared; T, thalamus; WM, white matter.
In larger animals, such as the cat, cooling can be used to inactivate a localized area, such that selected, or all, cortical layers can be reduced to $<20^{\circ} \mathrm{C}$, abolishing all neuronal spiking activity (Lomber et al., 1999). Projections to the IC primarily originate in layer $\mathrm{V}$ of auditory cortex, while projections to MGB arise from both layers V and VI (reviewed in Winer and Lee, 2007). With this in mind, it may be possible to selectively inactivate the upper layers of the cortex while leaving the activity in layer VI intact. Selective inactivation of the supragranular layers of cat primary visual cortex has previously been described (Schwark et al., 1986).

In the present study, we show physiological data from a series of experiments designed to test the limitations of cryoloop cooling as a model for examining corticofugal projections in the guinea pig auditory system. In smaller animals, such as the guinea pig, cooling is not so highly localized. Cryoloop cooling can be reliably used to reversibly inactivate core areas of auditory cortex, but not to produce layer-specific effects.

\section{MATERIALS AND METHODS ANIMALS}

All procedures were carried out in accordance with the Animals (Scientific Procedures) Act 1986, UK. Experiments were conducted on male and female pigmented guinea pigs (GPs; in-house colony) weighing $400-950 \mathrm{~g}$. In total, 27 GPs were included in this study; in some animals, more than 1 type of data was collected, and 11 of these GPs were used in descending systems experiments. For some of the parameters measured, the sample size is quite small. The inbred nature of the colony (minimizing anatomical 
variability) and the synergistic combination of techniques provided sufficient data to draw reliable conclusions. Attempts were made to use animals of similar weights when only two experimental animals were used. GPs were group-housed on a 12:12 h light:dark cycle, and food and water were freely available. Anesthesia was induced with urethane $\left(0.9-1.1 \mathrm{~g} \mathrm{~kg}^{-1}\right.$ in $20 \%$ solution, i.p.; SigmaAldrich, UK), and supplemented with Hypnorm (fentanyl citrate, $0.315 \mathrm{mg} \mathrm{ml}^{-1} /$ fluanisone, $10 \mathrm{mg} \mathrm{ml}^{-1}$, i.m., VetaPharma, UK) to maintain a constant state of areflexia. A single bolus injection of atropine sulfate $\left(0.06 \mathrm{mg} \mathrm{kg}^{-1}\right.$, s.c. $)$ was administered to suppress bronchial secretions.

\section{SURGERY}

In experiments where electrophysiological or temperature measurements were made, GPs were tracheotomized and respired with $100 \%$ oxygen to maintain normal end-tidal $\mathrm{CO}_{2}$ levels within a range of $28-38 \mathrm{~mm}$ of mercury. Core body temperature was monitored throughout and maintained to $38^{\circ} \mathrm{C} \pm 0.5^{\circ} \mathrm{C}$ using a homeothermic heating pad (Harvard Apparatus Ltd., Edenbridge, $\mathrm{UK}$ ) and a rectal probe. In thallium autometallography experiments GPs were not tracheotomized owing to the shorter nature of this procedure.

Animals were placed in a stereotaxic frame, with hollow plastic speculae replacing the ear bars, inside a sound-attenuating chamber. The bullae were vented on both sides using a polyethylene tube to equalize pressure across the tympanic membrane. The posterior fossa was opened to reduce respiratory pulsations of the brain. Craniotomies were performed over the right auditory cortex (7 mm diameter), MGB (5 mm diameter), and IC (4 mm diameter). The relatively large size of the craniotomy over auditory cortex in these experiments was necessary to accommodate the cooling loop. Alternatively, in experiments where temperature measurements were made across a large extent of neocortex, a single large craniotomy was performed over the right brain hemisphere. The dura mater was excised, and either single- or multi-electrode array recording electrodes, or a temperature-recording probe, were slowly lowered into either auditory cortex, surrounding regions of cortex, MGB, or IC; the exposed cortex was kept moist with intermittent application of $0.9 \%$ sodium chloride solution. In GPs where cochlea temperature was measured, the holes in the bullae were enlarged to accommodate a temperature-recording probe, as well as the venting tube.

\section{CORTICAL COOLING}

The right auditory cortex was periodically cooled using a custom-made 4-mm-diameter cooling loop (see Lomber et al., 1999 for a detailed description of the apparatus). Briefly, the cooling loop is constructed of a stainless steel tube $(0.25 \mathrm{~mm}$ lumen diameter) through which ethanol, cooled by dry ice, is pumped continuously. By altering the speed of the pump, the temperature of the cortex can be easily and quickly manipulated. The 4-mmdiameter cooling loop cools an area large enough to de-activate both core and belt areas (Wallace et al., 2000), a smaller loop than this may not reliably inactivate the belt region of auditory cortex. Cortical surface temperature was recorded by a thermocouple that protruded below the cooling loop. In the cat, a surface temperature of $2-3^{\circ} \mathrm{C}$ is sufficient to inactivate all cortical layers
(Lomber et al., 1999), i.e., input layers (III and IV), and layers from which descending projections originate (V and VI; Winer and Prieto, 2001).

\section{AUDITORY STIMULI}

Auditory stimuli were delivered diotically through sealed acoustic systems, composed of modified radio Shack 40-1377 tweeters joined via a conical section to a damped, 2.5-mm-diameter probe tube that fitted into the speculum. A search stimulus [generated by Tucker Davis Technologies (TDT) System 3; see Electrophysiology] was utilized to search for neuronal unit activity in the IC and MGB; this comprised a wideband noise (duration $50 \mathrm{~ms}$ ), gated on and off with cosine-squared ramps lasting $8 \mathrm{~ms}$, and a repetition period of $300 \mathrm{~ms}$. The use of multi-electrode arrays facilitated simultaneous recording across broad sections of the tonotopic axis. Consequently, search stimuli were selected to encompass a broad frequency range, thus maximizing identification of auditory-evoked neuronal singleunit activity. Single clicks were presented ( $50 \mu$ s duration, repeated at intervals of $400 \mathrm{~ms}$ ) binaurally to assess the degree of attenuation of auditory-evoked local field potentials and single-unit activity in auditory cortex.

\section{ELECTROPHYSIOLOGY}

Multiple extracellular single-units were filtered between $300 \mathrm{~Hz}$ and $3 \mathrm{kHz}$, and auditory-evoked field potentials were filtered between $60 \mathrm{~Hz}$ and $3 \mathrm{kHz}$. Field potentials were recorded from auditory cortex with a single shank, 16-channel "Michigan"-style silicon electrode. This silicon electrode array provided a depthwise recording span of $\sim 2 \mathrm{~mm}$, ideal for examining simultaneous changes in evoked potentials across the layers of auditory cortex. Field potentials represent the summed total of all localized presynaptic and postsynaptic activity from the surrounding neurones (Bullock, 1997). Auditory “click"-evoked potentials were recorded before cooling, and then at progressively lower cooling loop temperatures $\left(20,15,10\right.$, and $\left.2^{\circ} \mathrm{C}\right)$, and finally after a 20 -min recovery period ( $n=3 \mathrm{GPs}$ ). In another group of animals, single and multiunit recordings were made using glass-coated tungsten electrodes (configured as $1 \times 2,1 \times 4$, or $1 \times 8$ linear arrays). Tungsten electrodes ( 1-3 M $\Omega$ impedance) were attached to a single circuit board, with tips aligned and separated by $\sim 200 \mu \mathrm{m}$, and advanced simultaneously. All electrode arrays were connected via a TDT Medusa headstage amplifier (Alachua, FL, USA) to a TDT System 3; on-line data collection was facilitated with Brainware (software developed by J. Schnupp, University of Oxford, UK). Single- and multi-units were recorded in response to click stimuli in auditory cortex before, during cooling to $2^{\circ} \mathrm{C}$, and after cooling ( $n=11 \mathrm{GPs}$ ).

\section{TEMPERATURE MEASUREMENTS}

Changes in temperature were monitored in neocortex, IC, MGB, in ipsilateral and contralateral cochlea, and on the surface of the tentorium (a fold in the dura mater situated between the cerebellum and forebrain that contains draining venous blood). Measurements were made with an Omega 5SC-TT-KI-40 thermo-probe. This was composed of two wires ( $76 \mu \mathrm{m}$ diameter and plastic insulated) welded together to form a bead of $225 \mu \mathrm{m}$ diameter. Additional insulation was provided by a glass capillary, pulled on a conventional electrode pulling device, to within $500 \mu \mathrm{m}$ of the tip. 
In neocortex, measurements were made at the surface of the brain, and at depths of 500, 1000, and $1500 \mu \mathrm{m}$ (and also at intervening points in AI of two different GPs); for each animal, measurements were taken at $>60$ sites in auditory, visual, somatosensory, and frontal regions of the cortex, while the cooling loop temperature was maintained at $2^{\circ} \mathrm{C} \pm 1{ }^{\circ} \mathrm{C}(n=2 \mathrm{GPs})$.

Prior to collecting temperature data in the IC or the MGB, correct stereotaxic placement was first confirmed with single- and multi-unit recordings. Tungsten electrodes were subsequently replaced with the insulated thermo-probe, which was lowered to the same DV coordinates. The cooling loop was then turned on, and temperatures in either IC or MGB recorded at $1 \mathrm{~min}$-intervals for 20-25 min, while the loop was maintained at $2{ }^{\circ} \mathrm{C}(n=3 \mathrm{GPs}$ for each). This time-course protocol was also applied when measuring changes in cochlea (on the surface of the round window) and tentorium temperature ( $n=4$ GPs for ipsilateral cochlea; 1 GP for contralateral cochlea; 1 GP for tentorium).

\section{THALLIUM AUTOMETALLOGRAPHY}

Thallium uptake has not been studied in the GP brain before. Consequently, different amounts and administration routes for the thallium were used to try and optimize staining. The thallium was injected as a lipophilic compound by chelating it with sodium diethyldithiocarbamate (DDC) as recently described by Goldschmidt et al. (2010). This allows it to rapidly cross the blood-brain barrier and become distributed in the extracellular space. Surgical anesthesia was induced in experimental animals with urethane, the right auditory cortex was exposed, the dura was left intact and a cooling loop was placed over the cortex so that the surface of the cortex could be maintained at $2^{\circ} \mathrm{C}$ throughout the loading period and until the heart had stopped beating.

In three experimental animals, $8 \mathrm{ml}$ of a thallium suspension in $0.9 \%$ saline was prepared immediately before injection. This was formed by mixing $4 \mathrm{ml}$ of $0.8 \%$ thallium acetate with $4 \mathrm{ml}$ of $0.8 \%$ sodium DDC in a syringe and injecting it into the peritoneal cavity at two sites on the left/right abdomen. The animal was then killed by injecting an overdose of pentobarbitone (i.p.) 5, 7, or 9 min later.

In three other animals, the external jugular vein was cannulated before the craniotomy was performed, while in two control animals the vein was cannulated under anesthesia but no craniotomy was performed. A freshly prepared dose of thallium DDC consisting of $3 \mathrm{ml}$ of $0.2 \%, 3 \mathrm{ml}$ of $0.3 \%$, or $4 \mathrm{ml}$ of $0.25 \%$ suspension was slowly injected into the vein over a period of $3 \mathrm{~min}$ and flushed through with physiological saline. The animals were killed $5 \mathrm{~min}$ after the start of the injection by an overdose of pentobarbitone (i.v.).

Guinea pigs were then removed from the apparatus and immediately prepared for transcardial perfusion. GPs were perfused with $25 \mathrm{ml}$ of sulfide solution composed of $3.06 \mathrm{~g} \mathrm{NaH}_{2} \mathrm{PO}_{4}\left(1 \mathrm{H}_{2} \mathrm{O}\right)$ in $190 \mathrm{ml}$ distilled water combined with $10 \mathrm{ml}$ of a $6.5 \% \mathrm{Na}_{2} \mathrm{~S}$ solution with the $\mathrm{pH}$ brought to 7.4 with $\mathrm{NaOH}$. GPs were then perfused with $400 \mathrm{ml}$ of a solution containing $6.12 \mathrm{~g} \mathrm{NaH}_{2} \mathrm{PO}_{4}\left(1 \mathrm{H}_{2} \mathrm{O}\right)$ in $340 \mathrm{ml}$ of distilled water, $50 \mathrm{ml}$ of a $25 \%$ glutaraldehyde solution, and $10 \mathrm{ml}$ of a $6.5 \% \mathrm{Na}_{2} \mathrm{~S}$ stock solution, with $\mathrm{pH}$ adjusted to 7.4. Brains were removed from the skull and blocks cryoprotected for $36 \mathrm{~h}$ in $30 \%$ sucrose dissolved in $0.1 \mathrm{M}$ phosphate buffer $(\mathrm{pH}$ 7.4). Frozen sections were cut at $50 \mu \mathrm{m}$ in the coronal, sagittal, and horizontal planes and processed by the method described by
Goldschmidt et al. (2010). Densitometric measurements were made on corresponding structures from the two sides of the brain in coronal sections by using a Neurolucida (version 9) analysis system (MicroBrightField Inc., VT, USA). Mean values for pixel gray scale density were measured in circular samples (diameter $1.7 \mathrm{~mm}$ ) of digital images from auditory cortex, MGB, and IC of two control and two experimental brains.

\section{DATA ANALYSIS}

Temperature contour plots for neocortex were generated as follows: The exposed brain was photographed with a Nikon D70 dSLR camera, and each recording site manually marked on a printed image. This image was digitized and, along with the corresponding cortical temperatures, exported to Matlab. Contour plots were generated in Matlab and overlaid on original digital images.

Averaged auditory-evoked field potential responses to click stimuli were analyzed for changes in peak amplitude as the temperature of the cooling loop was reduced. To do this, the root mean squared (RMS) amplitude of averaged click-evoked potentials from each site on the silicon electrode, under each condition (pre-cooled, 20, $15,10,2^{\circ} \mathrm{C}$, and after cooling), were plotted and visually compared. This analysis was applied to data from multiple cycles of cooling in all experimental animals, except those used for thallium uptake. In addition, current source-density (CSD) analysis was applied to field potential data recorded across cortical layers. This method identifies current sinks and sources in the extracellular space (see Mitzdorf, 1985 for a comprehensive review). In this study, the CSD method was used in an attempt to more accurately determine changes in input layers and deeper layers containing the descending projection neurones.

Neuronal unit data from auditory cortex were examined for changes in firing rate between the "before cooling," "during cooling," and "recovery" conditions. Units were split into two groups according to the depth of recording: Units recorded at a depth of $<1000 \mu \mathrm{m}$ comprised the group for input layers, and $>1000 \mu \mathrm{m}$ for deep layers. Statistical comparisons were made with a two-way analysis of variance (ANOVA); where applicable, post hoc comparisons were made with Tukey's post hoc test.

\section{RESULTS}

In the present study, we aimed to determine the extent to which cooling deactivation spreads throughout the GP brain, and consequently test the viability of cryoloop cooling as a model for specific cortical inactivation in rodents. Cryoloop cooling has previously been validated for the cat, but to date has not been adequately addressed in smaller animals.

\section{TEMPERATURE CONTOURS AND NEURAL ACTIVITY IN AUDITORY CORTEX}

Temperatures were recorded at the cortical surface, and at depths through cortex of 500,1000, and $1500 \mu \mathrm{m}$, at a range of rostralcaudal and medial-lateral coordinates to the cooling loop. These sites sampled primary auditory cortex and other areas of the auditory, visual, and somatosensory regions. Temperatures recorded at $500 \mu \mathrm{m}$ below the pial surface, while the cooling loop (surface) temperature was maintained at $2 \pm 1^{\circ} \mathrm{C}$, are shown in Figures 1A,B ( $n=2$ GPs weighing 860 and $716 \mathrm{~g}$ ). Based on these measurements, 

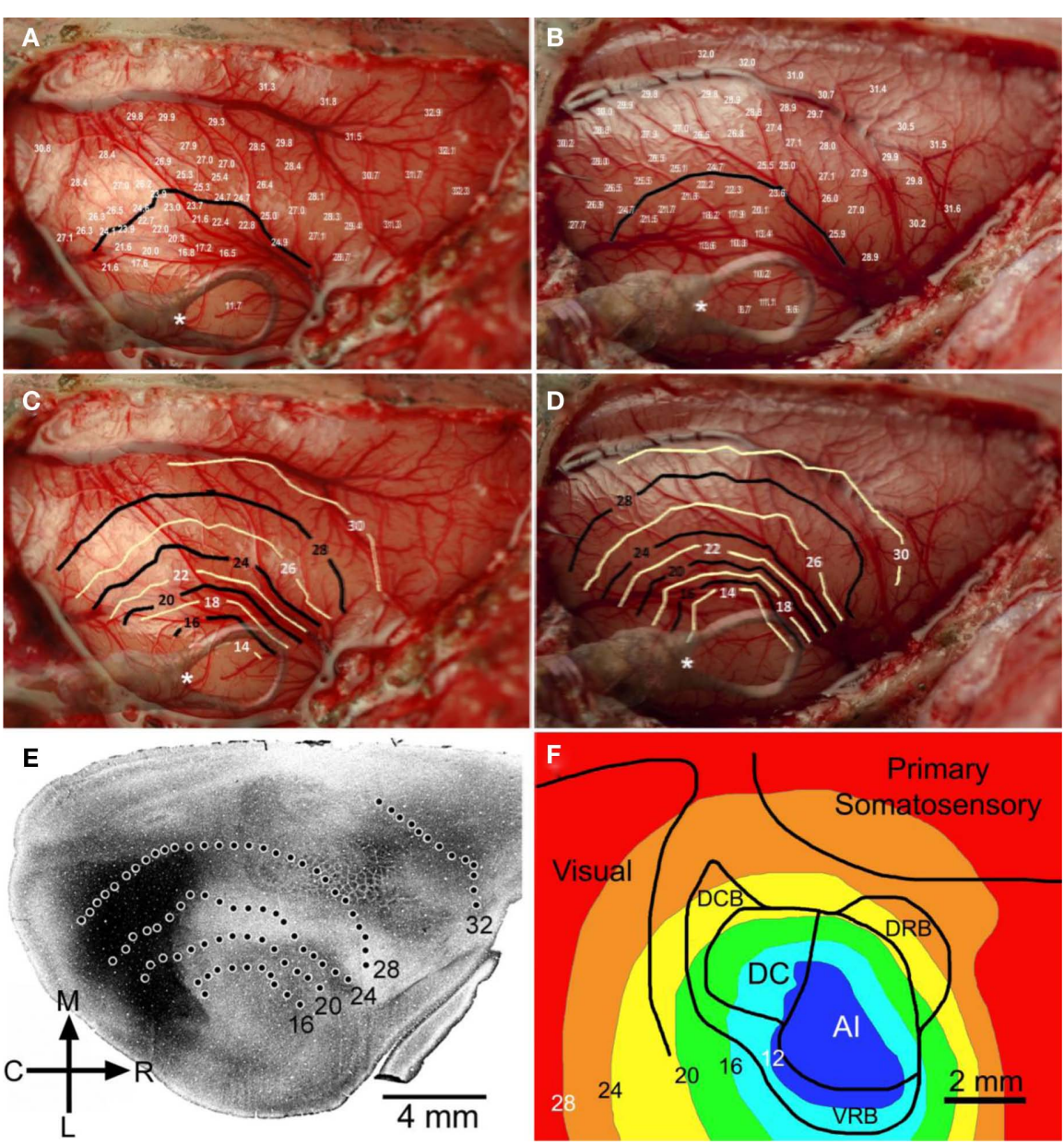

FIGURE 1 | Changes in cortical temperature. Temperature was sampled at multiple sites (>60) and depths $(500,1000$, and $1500 \mu \mathrm{m})$ across auditory, visual, and somatosensory regions of cortex. The temperature measurements taken at $500 \mu \mathrm{m}$ for two GPs \#1082 and GP\#1084 are shown in (A) and (B), along with $24^{\circ} \mathrm{C}$ contour lines generated for each animal. Contour plots were generated for each GP, shown in (C) and (D), at $500 \mu \mathrm{m}$ demonstrating the spread of cooling through cortical tissue. The position of the cooling loop was superimposed on each of these images and the position of the thermocouple monitoring cooling loop temperature was marked $\left(^{*}\right)$. (E) Cooling contours from the first GP [shown in $\mathbf{( A )}$ and $\mathbf{( C )}$ ] were overlaid on an image showing regional boundaries delineated by shading based on cytochrome oxidase staining (from Wallace et al., 2000). (F) Cooling contours from the second GP [shown in (B) and (D)] were overlaid on a schematic showing boundaries of auditory, somatosensory, and visual cortices. temperature contours were generated to illustrate the extent of cooling in the cortex (Figures 1C,D) and overlaid on actual photographs of the individual brains; contours revealed temperatures $<20^{\circ} \mathrm{C}$ up to $\sim 2.5 \mathrm{~mm}$ from the edge of the loop. Contour plots were also overlaid on anatomical/physiological maps of auditory cortex produced by this lab for comparison (Wallace et al., 2000). Temperatures $<20^{\circ} \mathrm{C}$ extended to the boundaries of outlying dorsocaudal belt and dorsorostral belt regions of auditory cortex that surround primary auditory cortex (A1 and DC). Moreover, cooling is not limited entirely to auditory cortex; temperatures were reduced in surrounding regions of cortex, potentially also affecting parts of visual cortex and somatosensory cortex (Figures 1E,F).

Neuronal evoked field potential activity was monitored depthwise across primary auditory cortex, at the center of the cooling loop. Cooling the surface of the cortex to $2^{\circ} \mathrm{C}$ abolished auditoryevoked field potentials in all cortical layers (Figure 2), in all animals tested ( $n=3$ GPs). CSD analysis revealed the presence of current sinks and sources that resemble CSD data shown by Mitzdorf (1985) derived from recordings in visual cortex. CSD plots did not reveal the presence of any layer-specific effects (Figure 2). Based on these observations, inactivation appeared to occur simultaneously in all layers; RMS local field potential amplitudes (Figure 3) exhibited a simultaneous decline as the temperature of the cooling loop was progressively decreased to $20,15,10$, and then to $2^{\circ} \mathrm{C}$.

Unit activity was recorded in 11 GPs with a single tungsten electrode positioned in the center of the cooling loop and at depths ranging from 245 to $2800 \mu \mathrm{m}$ in response to auditory "click" stimuli (Figures 4A,B; $n=13$ units). This showed that there was a large reduction or an abolition of evoked firing during the time when the cortical surface was cooled to $2^{\circ} \mathrm{C}$. Unit data were subdivided into two groups: (1) units recorded at depths of $<1000 \mu \mathrm{m}$, representative of recordings made from cortical layers II to IV, (2) units recorded at depths $>1000 \mu \mathrm{m}$, representative of activity in layers V and VI. Pooled neuronal firing rate data from these 


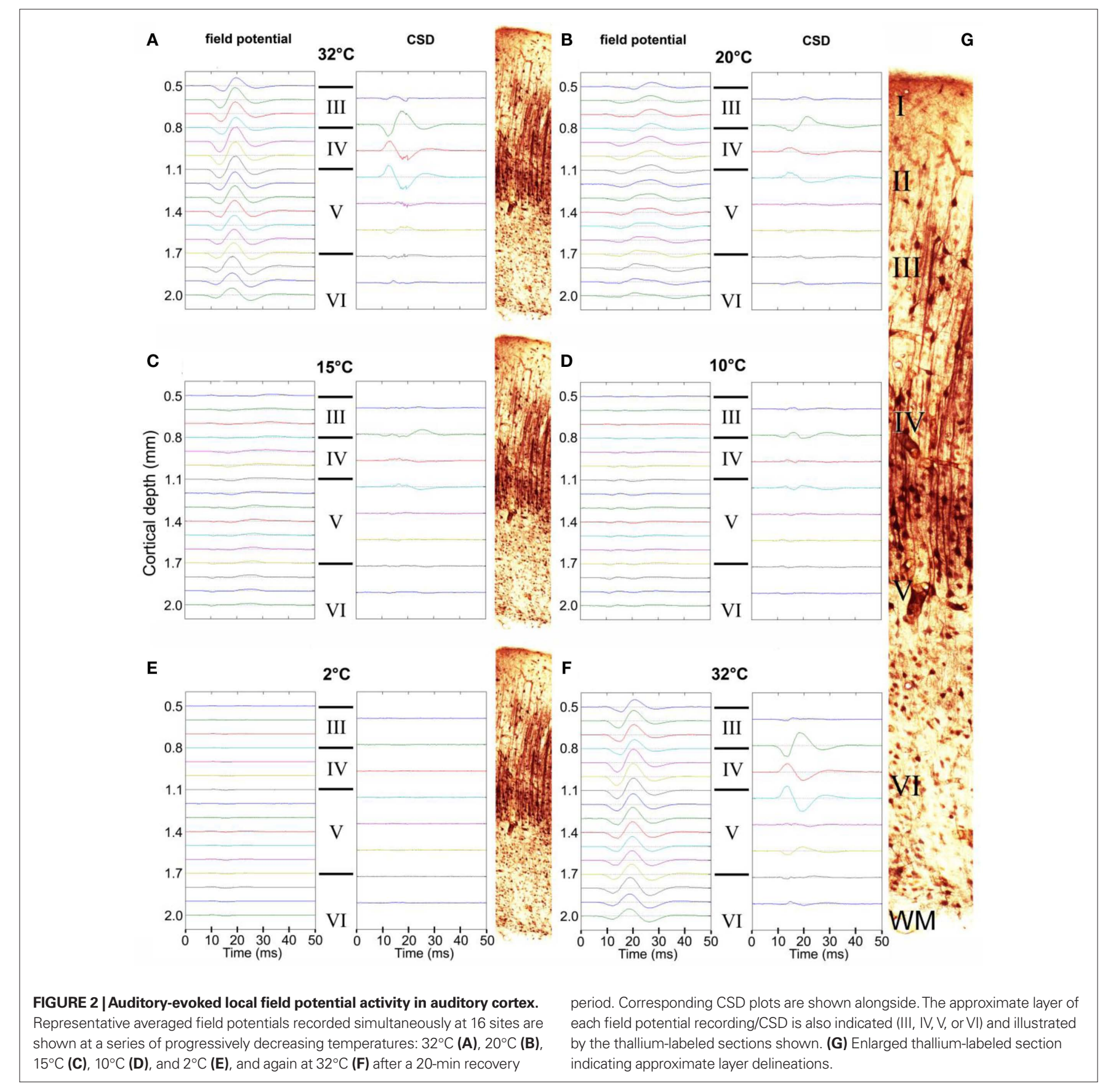

groups were compared with a two-way ANOVA before cooling, during cooling (cooling loop at $2{ }^{\circ} \mathrm{C}$ ), and after a 20 -min recovery period, revealing no statistically significant difference between layers II-IV and layers V-VI $\left[F_{(1,2)}=1.08, P=0.32\right]$. However, a significant effect of cooling compared with "before" and "after recovery" conditions was apparent $\left[F_{(2,2)}=10.98, P<0.01\right]$. No interaction between cortical layer grouping and time point was detected $\left[F_{(2,11)}=0.63, P=0.54\right]$. Further inspection revealed that, irrespective of cortical layer group, firing rate during cooling (mean firing rate $\pm S E M=0.37 \pm 0.20$ spikes s $^{-1}$ in layers II-IV; $0.40 \pm 0.23$ spikes $\mathrm{s}^{-1}$ in layers $\left.\mathrm{V}-\mathrm{VI}\right)$ was significantly lower $(P<0.01)$ than

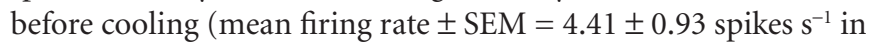

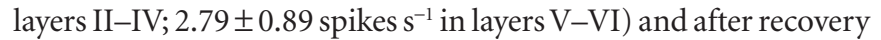

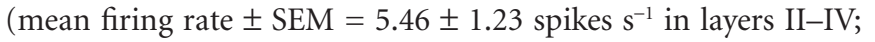
$3.56 \pm 1.88$ spikes s $^{-1}$ in layers $\mathrm{V}-\mathrm{VI}$ ).

Cortical temperature was also measured as a function of depth, while cooling loop temperature was maintained at $2{ }^{\circ} \mathrm{C}$ (Figure 5; $n=2$ GPs weighing 708 and $716 \mathrm{~g}$ ). Temperatures were recorded between the pial surface and a depth of $2500 \mu \mathrm{m}$ at the center of the loop. Variability in the cortex temperature measured with the probe did not exceed variability in surface temperature $\left( \pm 1^{\circ} \mathrm{C}\right)$ over a period of $10 \mathrm{~min}$. Thermoclines or temperature gradients were evident in both animals tested, ranging between $\sim 10$ and $\sim 15^{\circ} \mathrm{C}$ in GP \#1044, and between $\sim 12$ and $\sim 20^{\circ} \mathrm{C}$ in GP \#1084, up 
to a depth of $\sim 2000 \mu \mathrm{m}$. The thickness of auditory cortex varies between $\sim 2000$ and $2450 \mu \mathrm{m}$, dependent on position, although assessing thickness/recording depth is difficult due to the curvature of the brain. In high frequency regions cortex can be up to $2450 \mu \mathrm{m}$ thick, whereas in low frequency regions the cortex is closer to $2000 \mu \mathrm{m}$ thick (Wallace et al., 2000, 2008, 2011). Based

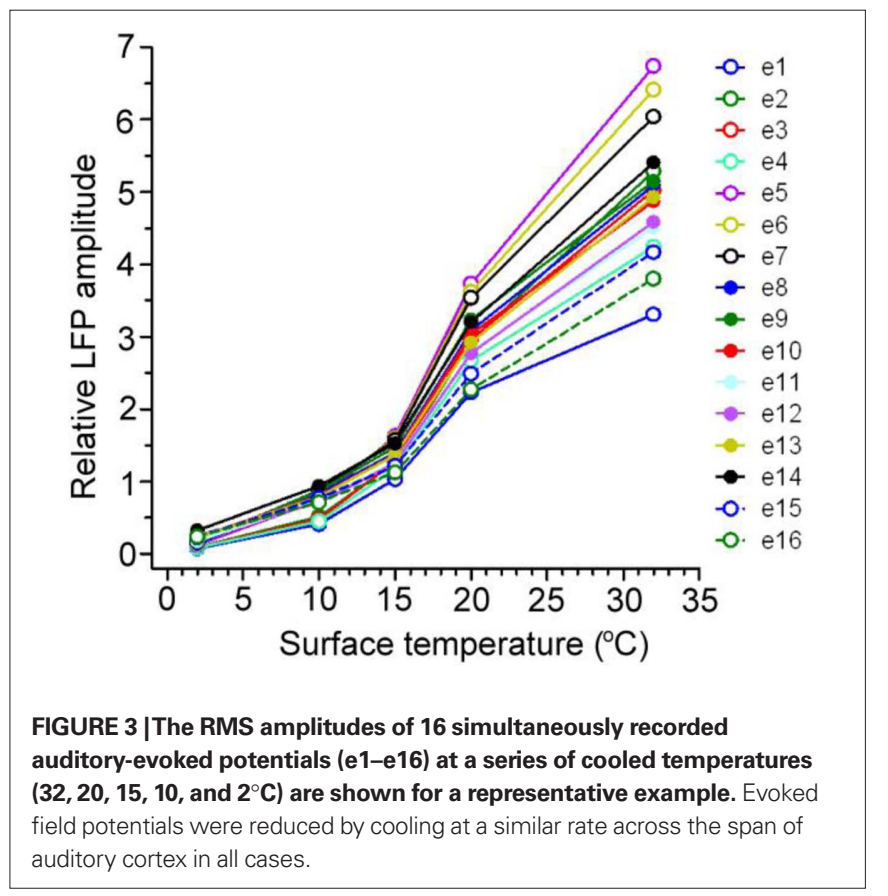

on this assertion, cortical temperature did not exceed $20^{\circ} \mathrm{C}$ in layers $\mathrm{I}-\mathrm{V}$ and a large proportion of layer $\mathrm{VI}$ in the auditory cortex of either animal.

\section{COOLING IN SUBCORTICAL AUDITORY NUCLEI}

Changes in temperature in other auditory nuclei were assessed, while auditory cortex was cooled to $2^{\circ} \mathrm{C}$, to rule out direct cooling effects at other stages of the auditory pathway. This included: IC, MGB, and ipsilateral and contralateral cochlea. Furthermore, the temperature at the junction of the superior sagittal and transverse sinuses at the internal border of the tentorium cerebelli was measured to give an indication of the temperature drop of the venous blood draining from the right hemisphere (Figure 6; $n=7 \mathrm{GPs}$ ). A decline from precooled temperatures of $\sim 4^{\circ} \mathrm{C}$ was detected in MGB (mean \pm SEM; from $34.8 \pm 0.9^{\circ} \mathrm{C}$ to $30.7 \pm 1.1^{\circ} \mathrm{C} ; n=3$ ) and IC (from $35.0 \pm 0.3^{\circ} \mathrm{C}$ to $31.0 \pm 0.9^{\circ} \mathrm{C} ; n=3$ ), shown in Figure $6 \mathrm{~A}$. Temperatures were reduced in the ipsilateral cochlea (from $34.4 \pm 0.3^{\circ} \mathrm{C}$ to $30.7 \pm 0.5^{\circ} \mathrm{C}$; $n=4$ ) and contralateral cochlea (from 34.7 to $32.6^{\circ} \mathrm{C} ; n=1$ ) from pre-cooled temperatures by $\sim 4$ and $\sim 2^{\circ} \mathrm{C}$, respectively (Figure 6B). Decreases in temperature in the cochlea are most likely due to cooled blood draining via the sinus that enters the internal jugular vein. To establish whether cold blood was responsible for changes at the cochlea, the temperature of the venous sinus at the tentorium was measured while cortex was cooled. A decline of $\sim 5^{\circ} \mathrm{C}$ in temperature (from 34.0 to $28.9^{\circ} \mathrm{C} ; n=1$ ) was observed (Figure 6B).

\section{THALLIUM IMAGING}

Thallium autometallography was used as a histological method to assess the extent of cooling deactivation. Auditory cortex was cooled to $2^{\circ} \mathrm{C}$ and thallium introduced by either an intravenous

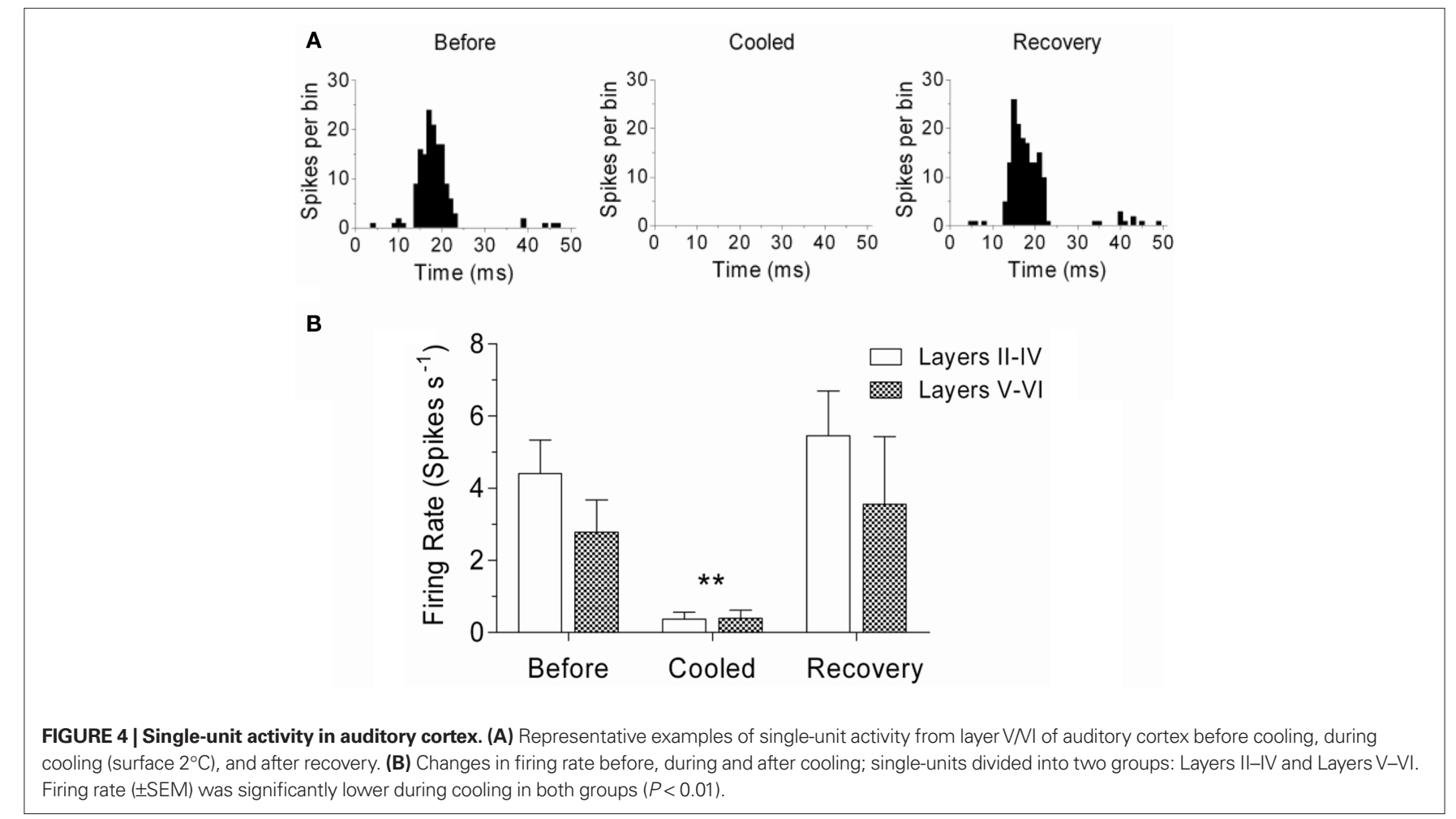




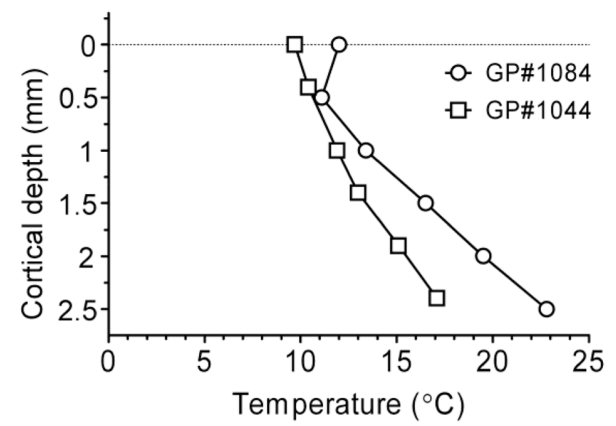

FIGURE 5 |Temperature recorded depth-wise across the span of auditory cortex in the center of the loop for two GPs during cooling (surface to $2^{\circ} \mathrm{C}$ ), illustrating the presence of thermoclines in auditory cortex.
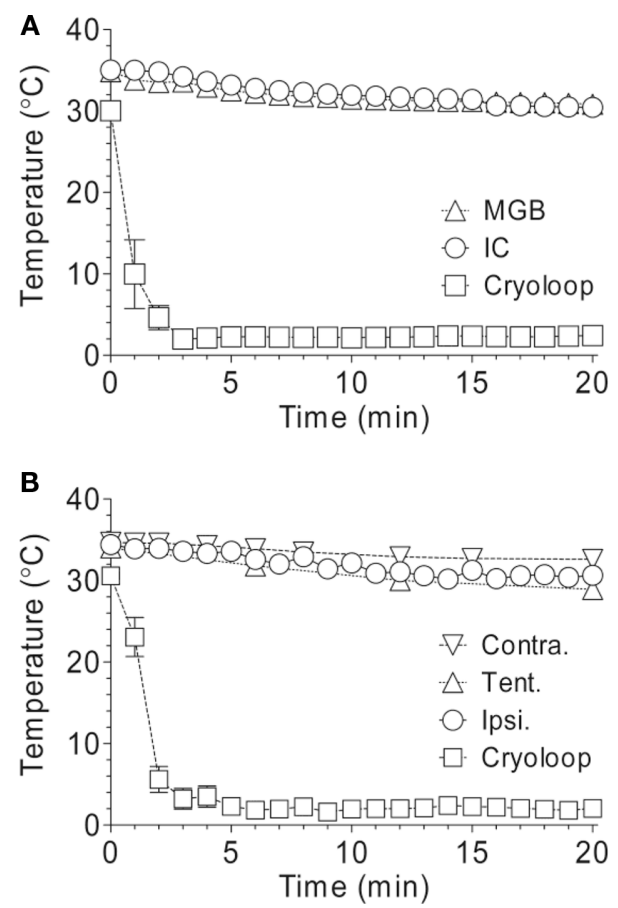

FIGURE 6 | Changes in temperature in subcortical auditory nuclei, ipsilateral and contralateral cochlea, and the tentorium. (A) Temperatures decreased by $\sim 4^{\circ} \mathrm{C}$ in the MGB and IC over a period of 20 min while the surface of auditory cortex was cooled to $2^{\circ} \mathrm{C}$. (B) Temperatures decreased in the ipsilateral and contralateral cochlea by $\sim 2$ and $\sim 4^{\circ} \mathrm{C}$, respectively, and by $\sim 5^{\circ} \mathrm{C}$ at the tentorium, while the cortex was cooled.

or intraperitoneal route. Thallium ions mimic potassium and are taken up from the extracellular space by neurones via the sodiumpotassium membrane pump. Cooling had profound effects on thallium staining in the cortex as illustrated in Figure 7. On the control (left) side there was clear neuronal uptake in all parts of the brain that led to darkly stained neurones (Figure 7A). In the neocortex staining was particularly prominent in the somata and apical dendrites of layer V pyramidal cells (Figure 7C), although other types of neurones were also darkly stained. By contrast, on the cooled (right) side a large part of the neocortex showed no neuronal staining at all (Figure 7B). The only staining on the cooled auditory cortex appeared to be on the endothelium of blood vessels (Figure 7D). This large asymmetry was not present at subcortical levels as the staining levels in the thalamus $(\mathrm{T})$ and midbrain were similar on the two sides (Figures 7A,B,E). In both the thalamus and IC staining was present mainly in individual neurones and their processes, but was also present in the walls of blood vessels (Figure 7F). The reduced thallium staining included all of the auditory cortex and much of the visual and somatosensory regions as well. The thallium staining gradually increased toward the margins of the neocortex and was close to control levels in the cingulate, lateral prefrontal, and entorhinal areas.

Although the thallium method is not a quantitative technique, it was appropriate to measure the staining intensity of corresponding structures from the two sides of the brain providing they had been identically treated and then to compare the values using paired $t$-tests. In the auditory cortex of the two control brains the ratio of densities between the right and left sides of the brain was 0.99. The values were not significantly different $\left[t_{(29)}=0.44 ; P=0.66\right]$. Similarly, the ratio for the IC was 0.94 and this was not significant $\left[t_{(43)}=1.81 ; P=0.08\right]$. However, there was a small but significant asymmetry in the MGB where the ratio was $1.15\left[t_{(31)}=6.1\right.$; $P<0.001]$. This meant that there was significantly less thallium staining in the right MGB of the controls. When similar measurements were taken from the two experimental brains, very different results were obtained. The density ratio between the right and left auditory cortex was 5.8 and this was highly significant $\left[t_{(26)}=15.85\right.$; $P<0.0001]$. The ratios between the two sides of the MGB was much smaller at 1.32 , but again this was significantly different $\left[t_{(11)}=3.05 ; P<0.05\right]$. This asymmetry of forebrain structures was no longer present in the IC, as the ratio between the two sides was 1.08 and was not significant $\left[t_{(28)}=1.67 ; P=0.11\right]$. Thus, the only region where there was a large difference between the control and experimental values was in the cortical region. This indicates that the cooling loop caused a large reduction in thallium uptake over a wide region of the neocortex that extended well beyond the borders of the auditory region.

\section{SUMMARY}

The aim of the present study was to validate cortical cooling as a method for reversibly inactivating neuronal activity in GPs. To this end, a number of parameters were assessed: Temperature was measured in neocortex and subcortical sites while the cooling loop was maintained at an "inactivating" temperature $\left(2^{\circ} \mathrm{C}\right)$, revealing variable temperature decline in auditory cortex, surrounding neocortex, IC, MGB, ipsilateral and contralateral cochlea, and the blood sinus at the tentorium. The "inactivating" temperature was determined by examining neural activity in auditory cortex, in response to "click" stimuli; cooling the surface to $2^{\circ} \mathrm{C}$ significantly attenuated evoked potentials and unit firing in primary auditory cortex. Thallium autometallography indicated that thallium uptake was greatly reduced by cooling in the cortex, but not in subcortical structures.

\section{DISCUSSION}

Cooling the surface of auditory cortex to $2^{\circ} \mathrm{C}$ reduced the temperature in all layers of primary auditory cortex to less than $20^{\circ} \mathrm{C}$, sufficient to attenuate auditory-evoked field potentials and unit 

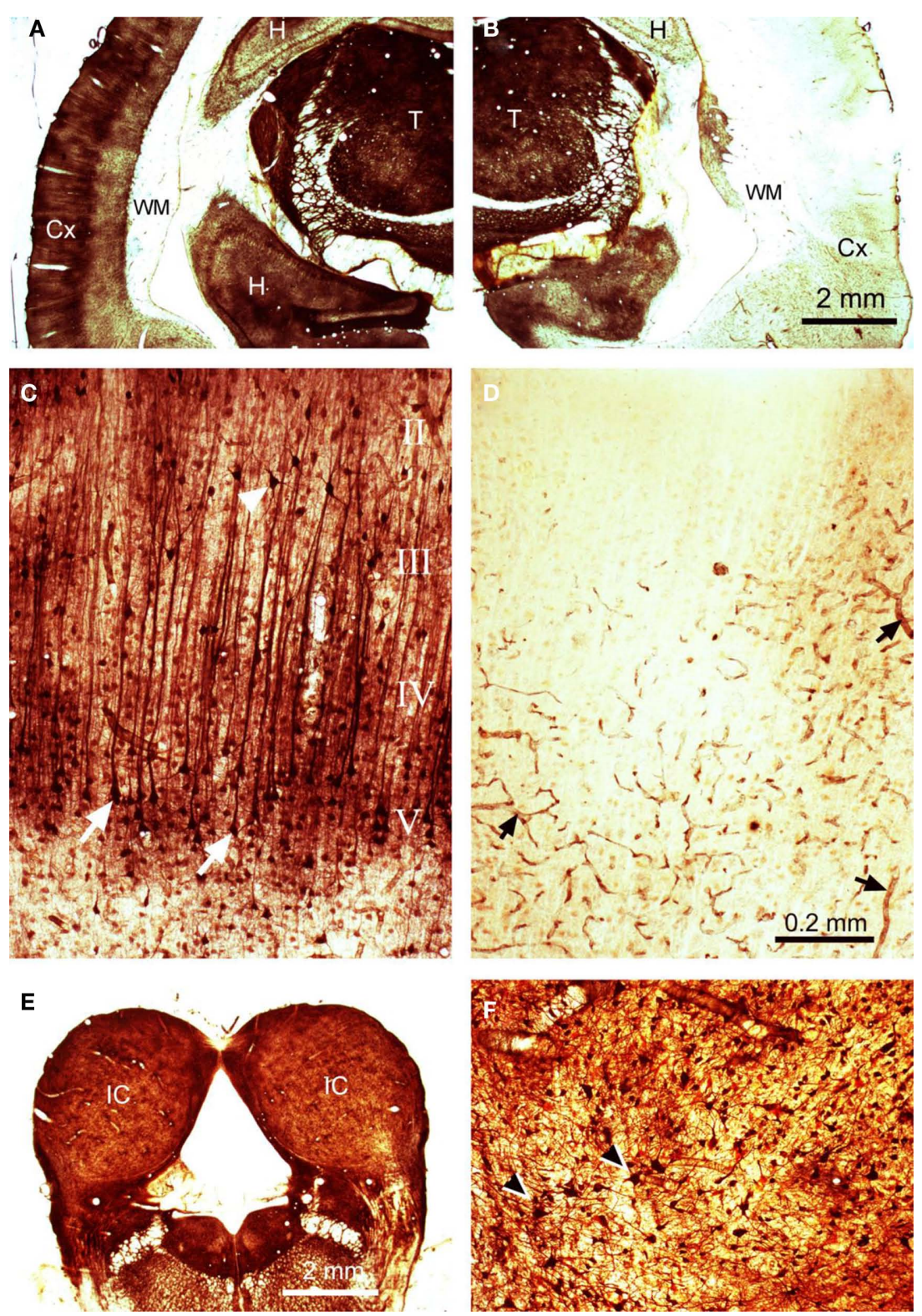

FIGURE 7 |Thallium autometallography. Coronal silver-stained sections are shown for (A) contralateral (left) cortex showing high levels of thallium uptake in the thalamus (T) and the superficial layers of the neocortex (CX) as well as parts of the hippocampus $(H)$, no staining was present in the white matter (WM), (B) cooled (right) cortex showing an almost complete abolition of thallium uptake in the neocortex, $\mathbf{( C )}$ left cortex at higher power showing prominent layer $\bigvee$ pyramidal cells with dark apical dendrites, (D) right cortex at higher power showing an absence of neuronal labeling but darkly stained blood vessels, (E) right and left IC showing similar levels of staining, $(\mathbf{F})$ stained neurones in the $\mathrm{IC}$ at a higher power. The magnification is the same for panels (A), (B), and (E) and for (C), (D), and (F). activity in all cortical layers. Lomber et al. $(1994,1999)$ found that neuronal function was diminished at temperatures of $<24^{\circ} \mathrm{C}$, and that at temperatures of $<20^{\circ} \mathrm{C}$ neuronal firing ceased in behaving cats. Inactivation of neural activity at temperatures $<20^{\circ} \mathrm{C}$ was also demonstrated in anesthetized cats (Michalski et al., 1993; Huang et al., 2007), and occurs due to blockade of synaptic activity below $20^{\circ} \mathrm{C}$ (Jasper et al., 1970; Bénita and Condé, 1972). The findings of the present study appear, in the main, to agree with data from previous studies, in terms of an inactivating temperature. Based on thermoclines measured in primary auditory cortex, a surface temperature of $2^{\circ} \mathrm{C}$ was required to reduce the temperature in all layers to $<20^{\circ} \mathrm{C}$, and this abolished neural activity.

Previous in vivo work found that cooling the surface of cat visual cortex to $10^{\circ} \mathrm{C}$ was sufficient to maintain temperatures between 10 and $20^{\circ} \mathrm{C}$, up to $\sim 2 \mathrm{~mm}$ from the edge of a silver "Peltier" cooling device. Deep cortical layers were therefore cooled to $10-20^{\circ} \mathrm{C}$ (Huang et al., 2007; Bardy et al., 2009). Earlier cooling studies, conducted in cat and primate cortex, indicated that a gradient effect of $5-6^{\circ} \mathrm{C} \mathrm{mm} \mathrm{m}^{-1}$ exists (Girard and Bullier, 1989; Michalski et al., 
1993). In these data, we observed a somewhat variable gradient (ranging from 1.5 to $6^{\circ} \mathrm{C} \mathrm{mm}^{-1}$ ) and that at surface temperatures $>2^{\circ} \mathrm{C}$, click-evoked local field potentials were still present, indicating some retention of neural function most likely attributable to thalamo-cortical volleys targeting input layers of auditory cortex. These differences may have been due to the proximity of the middle cerebral artery (MCA) to auditory cortex in the GP brain. The main part of the MCA is situated in the pseudo-Sylvian sulcus and lies just rostral to A1. It provides continuous perfusion of a relatively large volume of warm blood, potentially counteracting the cooling effect. Furthermore, the cooling device used in studies by Huang et al. (2007) and Bardy et al. (2009) differed from the cryoloop used in these experiments, presenting an additional confound to drawing comparisons between datasets.

Contrastingly, in vitro data obtained in slices from rat visual cortex showed that a local temperature of $<10^{\circ} \mathrm{C}$ was needed to completely abolish spike firing, although the amplitude of excitatory postsynaptic potentials was reduced in all neurones at temperatures $<20^{\circ} \mathrm{C}$. Furthermore, a degree of neuronal hyperexcitability, with regard to spike firing, was evident with smaller decreases in local temperature to $20-25^{\circ} \mathrm{C}$. This hyper-excitability was hypothesized to be caused by a part-depolarization of neurones, moving the resting membrane potential closer to a threshold for spike firing (Volgushev et al., 2000a,b). The explanation for differences between in vitro and in vivo data, in terms of inactivating temperature and neuronal hyper-excitability, is unclear. Increased excitability owing to decreased temperature could introduce a potential problem if cooling is to be used as a model for examining descending projections to subcortical auditory regions. However, the present data showed a decrease in temperature of only $\sim 4^{\circ} \mathrm{C}$ in the IC and MGB during cooling, and in neither case did the temperature fall below $30^{\circ} \mathrm{C}$. A decrease of this magnitude should not therefore be sufficient to contaminate data brought about by removal of descending input. Binaural effects in IC (Nakamoto et al., 2008, 2010) and both excitatory and inhibitory effects in MGB during cooling (Jones and Palmer, unpublished observations) also do not support neuronal hyper-excitability in this model. Taken together, the present data and previous studies (Lomber et al., 1994, 1999; Volgushev et al., 2000a,b; Huang et al., 2007; Bardy et al., 2009) indicate that a $4^{\circ} \mathrm{C}$ fall in temperature should not attenuate neuronal function in subcortical auditory nuclei.

The contrasting effects demonstrated by Volgushev et al. (2000a,b) and Lomber et al. (1999) do have implications when considering neocortex temperature contours. The $20^{\circ} \mathrm{C}$ contour in neocortex extended almost to the boundary of auditory cortex (based on anatomical estimates from Wallace et al., 2000). Core auditory areas (A1 and DC) exhibited temperatures of $<20^{\circ} \mathrm{C}$ and the majority of the belt auditory regions (dorsorostral and dorsocaudal belt regions) were also inactivated, at least in part $\left(<24^{\circ} \mathrm{C}\right)$. The $24^{\circ} \mathrm{C}$ contour did extend into other areas of cortex in some directions, and a direct cooling effect on neuronal function beyond the boundaries of auditory cortex cannot be ruled out. Paradoxically, given the findings of Volgushev et al. (2000a,b) neurones in belt regions and surrounding neocortex may be in a hyper-excitable state. However, given the similarities between this cryoloop protocol and that of Lomber et al. (1999) it seems sensible to make comparisons between these datasets, as opposed to in vitro data conducted with whole-slice-cooling. A caveat to using this model of inactivation in smaller animals, such as the GP, should therefore be that cooling the cortical surface to $2^{\circ} \mathrm{C}$ (found necessary to abolish activity across all layers) does not necessarily incur as localized an effect as it appeared to in the cat (Lomber et al., 1996, 1999, 2007).

The most probable mechanism for a spread of cooling is via blood flow. The MCA perfuses the temporal lobe with arterial blood. In order to test whether outgoing blood was affected during cooling, tentorium temperature was measured, revealing a decline of $\sim 5^{\circ} \mathrm{C}$. Reduced temperatures in the surrounding neocortex occurred caudally to a greater degree, coincident with the direction of blood flow from the MCA. Combined, these factors suggest that a large proportion of cooling spread is attributable to cooled blood flow. This effect limits the use of cooling as a model for distinguishing layer-specific effects; auditory-evoked potentials were simultaneously suppressed across cortical layers, implying that cooled blood (perfusing away from the MCA in a ventral direction) prevented selective cooling. Perfusion of cooled blood is also the most likely candidate for cochlear cooling $\left(\sim 4^{\circ} \mathrm{C}\right.$ ipsilateral and $\sim 2^{\circ} \mathrm{C}$ contralateral $)$. In humans, venous blood collects at the internal edge of the tentorium before traveling along the sigmoid sinus, which sits in the sigmoid sulcus on the surface of the petrous temporal bone that surrounds the cochlea, and then drains into the jugular foramen. A similar route is taken by venous blood in the guinea pig and this flow of cooled blood over the thin spongy bone of the bulla appears to be the cause of cochlear cooling. The $4^{\circ} \mathrm{C}$ reduction in temperature at the round window is probably sufficient to alter cochlear function by a small amount (Butler et al., 1960). However, an altered cochlear output alone does not explain the effects of cooling auditory cortex on processes at the level of the IC (Nakamoto et al., 2008, 2010).

We attempted to use the uptake of thallium ions as a means of mapping out the extent of reduced neuronal activity. However, we found that the area of reduced thallium uptake extended beyond the area of cortex marked by the $30^{\circ}$ contour. At this distance from the cooling loop there should not have been any reduction in neuronal function and yet thallium uptake was still reduced. This implied that the thallium levels were not only related to neuronal activity but were also affected by some other change. The reason for this is not obvious but may involve a decreased blood flow falling below a level where there is sufficient thallium transported to form stable thallous sulfide nanocrystals that can then be amplified during silver development. Thallium uptake appears to be very sensitive to blood flow effects as well as cooling. This made the finding of only small changes at subcortical levels all the more striking. It implies that there had been little change in the blood supply to the thalamus and midbrain on the cooled side and was consistent with the temperature measurements. This further implies that there was little effect on neuronal function. The significance of the asymmetry in thallium uptake in the MGB prior to cooling is unclear. It may reflect a small asymmetry in the auditory structures of the forebrain but could also be due to fluctuations in the blood supply caused by the deep anesthesia. 
There are a multitude of available techniques and tools for neuronal inactivation, some reversible some not, and each technique is accompanied by certain limitations (reviewed by Lomber, 1999). Inactivation techniques vary in duration of the inactivation and time to recovery, size and subsequent regional localization of inactivation, and whether there are functional compensations. Cooling represents a technique that offers complete reversibility, and benefits from a highly controllable duration of inactivation and rapid recovery, factors that contribute to the absence of functional compensations (Lomber, 1999). Cooling inactivation is a well-established technique used for studying the dynamics of neuronal signaling pathways. It has been utilized extensively to study

\section{REFERENCES}

Bardy, C., Huang, J. Y., Wang, C., Fitzgibbon T., and Dreher B. (2009). 'Top-down' influences of ipsilateral or contralateral postero-temporal visual cortices on the extra-classical receptive fields of neurons in cat's striate cortex. Neuroscience 158, 951-968.

Bénita,M., and Condé, H. (1972). Effects of cooling upon conduction and synaptic transmission. Brain Res. 36, 133-151.

Bullock, T. H. (1997). Signals and signs in the nervous system: the dynamic anatomy of electrical activity is probably information-rich. Proc. Natl. Acad. Sci. U.S.A. 94, 1-6.

Butler, R. A., Konishi, T., and Fernandez, C. (1960). Temperature coefficients of cochlear potentials. Am. J. Physiol. 199, 688-692.

Coomes Peterson, D., and Schofield, B. R. (2007). Projections from auditory cortex contact ascending pathways that originate in the superior olive and inferior colliculus. Hear. Res. 232, 67-77.

Feliciano, M., and Potashner, S. J. (1995). Evidence for a glutamatergic pathway from the guinea pig auditory cortex to the inferior colliculus. J. Neurochem. 65, 1348-1357.

Girard, P., and Bullier, J. (1989). Visual activity in area V2 during reversible inactivation of area 17 in the macaque monkey. J. Neurophysiol.62, 1287-1302.

Goldschmidt, J., Wanger, T., Engelhorn, A., Friedrich, H., Happel, M., Ilango, A., Engelmann, M., Stuermer, I. W., Ohl, F. W., and Scheich, H. (2010). High-resolution mapping of neuronal activity using the lipophilic thallium chelate complex TIDDC: protocol and validation of the method. Neuroimage 49, 303-315.

He, J. (2003). Corticofugal modulation of the auditory thalamus. Exp. Brain Res. 153, 579-590.

Huang, J. Y., Wang, C., and Dreher, B. (2007). The effects of reversible inactivation of postero-temporal visual cortex on neuronal activities in cat's area 17. Brain Res. 1138, 111-128.

Jasper, H. H., Shacter, D. G., and Montplaisir, J. (1970). The effect of local cooling upon spontaneous and evoked electrical activity of cerebral cortex. Can . J. Physiol. Pharmacol. 48, 640-652.

Kelly, J. P., and Wong, D. (1981). Laminar connections of the cat's auditory cortex. Brain Res. 212, 1-15.

Liu, X., Yan, Y., Wang, Y., and Yan, J. (2010). Corticofugal modulation of initial neural processing of sound information from the ipsilateral ear in the mouse. PLoS ONE 5, e14038. doi: 10.1371/journal.pone.0014038

Lomber, S. G. (1999). The advantages and limitations of permanent or reversible deactivation techniques in the assessment of neural function. J. Neurosci. Methods 86, 109-117.

Lomber, S. G., Cornwell, P., Sun, J. S., MacNeil,M.A., and Payne, B. R. (1994). Reversible inactivation of visual processing operations in middle suprasylvian cortex of the behaving cat. Proc. Natl. Acad. Sci. U.S.A. 91, 2999-3003.

Lomber, S. G., Malhotra, S., and Hall, A. J. (2007). Functional specialization in non-primary auditory cortex of the cat: areal and laminar contributions to sound localization. Hear. Res. 229,31-45. Lomber, S. G., Payne, B. R., and Cornwell, P. (1996). Learning and recall of form discriminations during reversible cooling deactivation of ventralposterior suprasylvian cortex in the cat. Proc. Natl. Acad. Sci. U.S.A. 93, 1654-1658.

Lomber, S. G., Payne, B. R., and Horel, J. A. (1999). The cryoloop: an adaptable reversible cooling deactivation method for behavioural or electrophysiological assessment of neural function. J. Neurosci. Methods 86, 179-194.

Luo, F., Wang, Q., Kashani, A., and Yan, J. (2008). Corticofugal modulation of initial sound processing in the brain. J. Neurosci. 28, 11615-11621.

Michalski, A., Wimborne, B. M., and Henry, G. H. (1993). The effect of reversible cooling of cat's primary visual cortex on the responses of area 21a neurons. J. Physiol. 466, 133-156.

Mitzdorf, U. (1985). Current source-density method and application in cat cerebral cortex: investigation of evoked

visual and auditory processing in cats (Schwark et al., 1986; Villa and Abeles, 1990; Lomber et al., 1999; Wang et al., 2007; Bardy et al., 2009), and in rats and guinea pigs (Villa et al., 1999; Nakamoto et al., 2008).

In short, cooling is an effective and reversible method of inactivation but is not limitation-free, nor is it as localized in the GP as it is in the cat. This is more than likely due to blood perfusion effects. The temperature in subcortical auditory nuclei was reduced, but, importantly, this was unlikely to substantially alter neuronal activity. To conclude, cooling inactivation remains a viable technique in smaller animals, but may not produce the localized effect achievable in larger species, such as the cat.

potentials and EEG phenomena. Physiol. Rev. 65, 37-100.

Nakamoto, K. T., Jones, S. J., and Palmer,A. R. (2008). Descending projections from auditory cortex modulate sensitivity in the midbrain to cues for spatial position. J. Neurophysiol. 99, 2347-2356.

Nakamoto, K. T., Shackleton, T. M., and Palmer, A. R. (2010). Responses in the inferior colliculus of the guinea pig to concurrent harmonic series and the effect of inactivation of descending controls. J. Neurophysiol. 103, 2050-2061.

Saldaña, E., Feliciano, M., and Mugnaini, E. (1996). Distribution of descending projections from primary auditory neocortex to inferior colliculus mimics the topography of intracollicular projections. J. Comp. Neurol. 371, 15-40.

Schwark, H. D., Malpeli, J. G., Weyand, T. G., and Lee, C. (1986). Cat Area 17. II. Response properties of infragranular layer neurons in the absence of supragranular layer activity. J. Neurophysiol. 56, 1074-1087.

Villa, A. E. P., and Abeles, M. (1990) Evidence for spatiotemporal firing patterns within the auditory thalamus of the cat. Brain Res. 509, 325-327.

Villa, A. E. P., Tetko, I. V., Dutoit, P., De Ribaupierre, Y., and De Ribaupierre, F. (1999). Corticofugal modulation of functional connectivity within the auditory thalamus of rat, guinea pig and cat revealed by cooling deactivation. J. Neurosci. Methods 86, 161-178.

Volgushev,M.,Vidyasagar, T.R., Chistiakova, M., and Eysel, U. T. (2000a). Synaptic transmission in the neocortex during reversible cooling. Neuroscience 98,9-22.

Volgushev, M., Vidyasagar, T.R., Chistiakova, M., Yousef, T., and Eysel, U. T. (2000b). Membrane properties and spike generation in rat visual cortical cells during reversible cooling. J. Physiol. 522, 59-76.

Wallace, M. N., Coomber, B., Sumner, C. J., Grimsley, J.M., Shackleton, T.M., and Palmer, A. R. (2011). Location of cells giving phase-locked responses to pure tones in the primary auditory cortex. Hear. Res. 274, 142-151.

Wallace, M. N., and Palmer, A. R. (2008). Laminar differences in the response properties of cells in the primary auditory cortex. Exp. Brain Res. 184, 179-191.

Wallace, M. N., Rutkowski, R. G., and Palmer,A. R. (2000). Identification and localisation of auditory areas in guinea pig cortex. Exp. Brain Res. 132, 445-456.

Wang, C., Waleszczyk, W. J., Burke, W., and Dreher, B. (2007). Feedback signals from the cat's area 21a enhance orientation selectivity of area 17 neurons. Exp. Brain Res. 182, 479-490.

Weedman, D. L., and Ryugo, D. K. (1996a). Projections from auditory cortex to the cochlear nucleus in rats: synapses on granule cell dendrites. J. Comp. Neurol. 371, 311-324.

Weedman, D. L., and Ryugo, D. K. (1996b). Pyramidal cells in primary auditory cortex project to cochlear nucleus in rat. Brain Res. 706, 97-102.

Winer, J. A., and Lee, C. C. (2007). The distributed auditory cortex. Hear. Res. 229, 3-13.

Winer, J. A., and Prieto, J. J. (2001). Layer $\mathrm{V}$ in cat primary auditory cortex (AI): cellular architecture and identification of projection neurons. J. Comp. Neurol. 434, 379-412.

Conflict of Interest Statement: The authors declare that the research was conducted in the absence of any commercial or financial relationships that could be construed as a potential conflict of interest.

Received: 24May 2011;paperpending published:03 June 2011; accepted:09 June 2011; published online: 21 June 2011.

Citation: Coomber B, Edwards D, Jones SJ, Shackleton TM, Goldschmidt J, Wallace $M N$ and Palmer AR (2011) Cortical inactivation by cooling in small animals. Front. Syst. Neurosci. 5:53. doi: 10.3389/ fnsys.2011.00053

Copyright (C) 2011 Coomber, Edwards, Jones, Shackleton, Goldschmidt, Wallace and Palmer. This is an open-access article subject to a non-exclusive license between the authors and Frontiers Media SA, which permits use, distribution and reproduction in other forums, provided the original authors and source are credited and other Frontiers conditions are complied with. 